\title{
On the 2PN Pericentre Precession in the General Theory of Relativity and the Recently Discovered Fast-Orbiting S-Stars in Sgr $A^{*}$
}

\section{Lorenzo Iorio}

check for

updates

Citation: Iorio, L. On the 2PN

Pericentre Precession in the General

Theory of Relativity and the Recently

Discovered Fast-Orbiting S-Stars in

Sgr A*. Universe 2021, 7, 37.

https://doi.org/10.3390/

universe7020037

Academic Editor: Philippe Jetzer

Received: 1 January 2021

Accepted: 2 February 2021

Published: 4 February 2021

Publisher's Note: MDPI stays neutral with regard to jurisdictional clai$\mathrm{ms}$ in published maps and institutional affiliations.

Copyright: (C) 2021 by the author. Licensee MDPI, Basel, Switzerland. This article is an open access article distributed under the terms and conditions of the Creative Commons Attribution (CC BY) license (https:// creativecommons.org/licenses/by/ $4.0 /)$.
Ministero dell'Istruzione, dell'Università e della Ricerca (M.I.U.R.)-Istruzione, Viale Unità di Italia 68, 70125 Bari (BA), Italy; lorenzo.iorio@libero.it

\begin{abstract}
Recently, the secular pericentre precession was analytically computed to the second postNewtonian (2PN) order by the present author with the Gauss equations in terms of the osculating Keplerian orbital elements in order to obtain closer contact with the observations in astronomical and astrophysical scenarios of potential interest. A discrepancy in previous results from other authors was found. Moreover, some of such findings by the same authors were deemed as mutually inconsistent. In this paper, it is demonstrated that, in fact, some calculation errors plagued the most recent calculations by the present author. They are explicitly disclosed and corrected. As a result, all of the examined approaches mutually agree, yielding the same analytical expression for the total $2 \mathrm{PN}$ pericentre precession once the appropriate conversions from the adopted parameterisations are made. It is also shown that, in the future, it may become measurable, at least in principle, for some of the recently discovered short-period S-stars in Sgr A*, such as S62 and S4714.
\end{abstract}

Keywords: general relativity and gravitation; celestial mechanics

\section{Introduction}

The analytical calculation of the secular second post-Newtonian $(2 \mathrm{PN})^{1}$ pericentre ${ }^{2}$ precession $\dot{\omega}^{2 \mathrm{PN}}$ of a gravitationally bound two-body system made of two mass monopoles $M_{\mathrm{A}}, M_{\mathrm{B}}$ with the perturbative Gauss equations for the variation of the osculating Keplerian orbital elements (e.g., [5,6]) was the subject of Iorio [7]. For the sake of simplicity, the test particle limit will be considered in most of this paper. In the following, $c$ is the speed of light in vacuum, $\mu \doteq G M$ is the gravitational parameter of the primary whose mass is $M, G$ is the Newtonian constant of gravitation, $\mathbf{v}, \mathrm{v}_{r}, \mathrm{v}$ are the test particle's velocity, radial velocity, and speed, respectively, $r$ is the test particle's distance from the primary, $\hat{r}$ is the position unit vector of the test particle with respect to the primary, $f_{0}, a, e$ are [8] the osculating numerical values of the true anomaly, semimajor axis, and eccentricity, respectively, at the same arbitrary moment of time $t_{0}$, and $n_{\mathrm{b}}=\sqrt{\mu / a^{3}}$ is the osculating mean motion.

The expression for the total $2 \mathrm{PN}$ pericentre precession derived by Iorio [7] consists of the sum of three contributions. The first one, dubbed as "direct", is ([7], Equation (8)):

$$
\dot{\omega}_{\mathrm{dir}}^{2 \mathrm{PN}}=\frac{n_{\mathrm{b}} \mu^{2}\left(28-e^{2}\right)}{4 c^{4} a^{2}\left(1-e^{2}\right)^{2}}
$$

arising straightforwardly from the $2 \mathrm{PN}$ acceleration

$$
A^{2 \mathrm{PN}}=\frac{\mu^{2}}{c^{4} r^{3}}\left[\left(2 \mathrm{v}_{r}^{2}-\frac{9 \mu}{r}\right) \hat{\boldsymbol{r}}-2 \mathrm{v}_{r} \mathbf{v}\right] .
$$

1 For an overview of post-Newtonian (PN) theory and its applications, see, e.g., Blanchet [1], Will [2,3] and references therein.

2 The precession of the pericentre is one of the post-Keplerian $(\mathrm{pK})$ parameters that are used in testing gravitational theories in astrophysical binary systems containing at least one compact object; see, e.g., Zhang et al. [4]. 
There are also two further contributions, labeled as "mixed" or "indirect". They account for the fact that, when the Gauss equation for the rate of change of the pericentre induced by the first post-Newtonian (1PN) acceleration,

$$
A^{1 \mathrm{PN}}=\frac{\mu}{c^{2} r^{2}}\left[\left(\frac{4 \mu}{r}-\mathrm{v}^{2}\right) \hat{r}+4 \mathrm{v}_{r} \mathbf{v}\right]
$$

is averaged over one orbital period $P_{\mathrm{b}}$, the latter one has to be considered as the time interval between two consecutive crossings of the moving pericentre. Moreover, the instantaneous shifts of the other orbital elements due to Equation (3) itself are also to be taken into account when the orbital average is performed. Both such effects contribute the total pericentre precession to the 2PN level. The first indirect effect yields ([7], Equation (14)):

$$
\dot{\omega}_{\text {indir }}^{2 \mathrm{PN}(\mathrm{I})}=\frac{n_{\mathrm{b}} \mu^{2}\left(9+37 e^{2}+e^{4}\right)}{2 c^{4} a^{2} e^{2}\left(1-e^{2}\right)^{2}},
$$

while the second indirect contribution reads ([7], Equation (22)):

$$
\dot{\omega}_{\text {indir }}^{2 \mathrm{PN}(\mathrm{II})}=-\frac{n_{\mathrm{b}} \mu^{2}\left\{9-87 e^{2}-136 e^{4}+19 e^{6}-6 e^{3}\left[\left(34+26 e^{2}\right) \cos f_{0}+15 e \cos 2 f_{0}\right]\right\}}{2 c^{4} e^{2} a^{2}\left(1-e^{2}\right)^{3}} .
$$

Thus, the sum of Equations (1), (4), and (5) gives the total 2PN pericentre precession:

$$
\dot{\omega}_{\mathrm{tot}}^{2 \mathrm{PN}}=\frac{3 n_{\mathrm{b}} \mu^{2}\left[86+57 e^{2}-13 e^{4}+8 e\left(17+13 e^{2}\right) \cos f_{0}+60 e^{2} \cos 2 f_{0}\right]}{4 c^{4} a^{2}\left(1-e^{2}\right)^{3}} .
$$

Iorio [7] compared his results with those by Kopeikin and Potapov [9], who used the perturbative approach relying upon the Gauss equations as well, and those by Damour and Schafer [10], which were obtained with the Hamilton-Jacobi method. A discrepancy in the results of these authors was found, since Iorio [7] claimed that the total 2PN pericentre precession inferred by (Kopeikin and Potapov [9] Equation (5.2)) can be cast into the form ([7], Equation (53)):

$$
\dot{\omega}_{\mathrm{tot}}^{2 \mathrm{PN}}=\frac{3 n_{\mathrm{b}} \mu^{2}\left(2+e^{2}-32 e^{2} \cos f_{0}\right)}{4 c^{4} a^{2}\left(1-e^{2}\right)^{2}} .
$$

Actually, a typo occurred in (Iorio [7] Equation (53)), since the correct expression reads:

$$
\dot{\omega}_{\mathrm{tot}}^{2 \mathrm{PN}}=\frac{3 n_{\mathrm{b}} \mu^{2}\left(2+e^{2}-32 e \cos f_{0}\right)}{4 c^{4} a^{2}\left(1-e^{2}\right)^{2}} ;
$$

see Section 2. Equation (8) was not explicitly shown by Kopeikin and Potapov [9]; it was mistakenly reproduced in (Kopeikin [11] Equation (44)) by writing $-32 \cos f_{0}$ in the numerator.

Furthermore, Iorio [7] claimed that (Damour and Schafer [10] Equation (3.12)), which was demonstrated to be coincident with Equation (8) once the aforementioned typo is taken into account, and (Damour and Schafer [10] Equation (5.18)) would be mutually inconsistent.

Here, it will be proven that, actually, a mere calculation error occurred in the derivation of $\dot{\omega}_{\text {indir }}^{2 \mathrm{PN}(\mathrm{II})}$ by Iorio [7], which prevented the author from obtaining Equation (8) instead of the incorrect Equation (6). Once this error is corrected and the typo in Equation (7) is removed, both the approaches by Iorio [7] and Kopeikin and Potapov [9], which differ in how they obtain just $\dot{\omega}_{\text {indir }}^{2 \mathrm{PN}}$, agree, yielding the same total $2 \mathrm{PN}$ pericentre precession as that of Equation (8). Moreover, it will be shown that the alleged inconsistency of Equations (3.12) and (5.18) by Damour and Schafer [10] is, in fact, also due to another error 
by Iorio [7], as correctly pointed out by Kopeikin [11]. For other examples of different parameterisations used in calculating the 2PN precession, see, e.g., Amaro-Seoane [12].

To the benefit of the reader, it is noted that Kopeikin and Potapov [9], Damour and Schafer [10] usually dealt with the fractional pericentre advance per orbit, i.e., $\Delta \omega / 2 \pi$; in order to obtain the corresponding precession, it is sufficient to multiply it by $n_{\mathrm{b}}$.

The paper is organised as follows.

In Section 2 , the calculation error in working out $\dot{\omega}_{\text {indir }}^{2 \mathrm{PN}(\mathrm{II})}$ is explicitly disclosed and corrected, and Equation (8) is obtained. Section 3 is devoted to showing, independently from Kopeikin [11], that Equations (3.12) and (5.18) of Damour and Schafer [10] are, in fact, mutually consistent, with both yielding the same total 2PN pericentre precession as Equation (8). Some aspects of the presence of $f_{0}$ in Equation (8) are discussed in Section 4. Section 5 is devoted to calculating the $2 \mathrm{PN}$ pericentre precession for some of the recently discovered fast-orbiting S-stars [13] in the Galactic Center (GC) at Sgr A*. Section 6 summarises the present findings and offers concluding remarks.

\section{Disclosing and Correcting the Error for $\dot{\omega}_{\text {indir }}^{2 \mathrm{PN}}$ (II)}

In Iorio [7], it turned out that Equations (1) and (4) agreed with the corresponding calculation by Kopeikin and Potapov [9], despite that these authors neither recurred to the schematisation by Iorio [7] nor explicitly displayed their intermediate results.

Instead, Iorio [7] realised that the discrepancy between his results and those by Kopeikin and Potapov [9] resides in $\dot{\omega}_{\text {indir }}^{2 \mathrm{PN}(\mathrm{II})}$, i.e., in the part of the indirect precession arising from the fact that the semimajor axis and the eccentricity do change instantaneously during an orbital revolution due to Equation (3) according to

$$
\begin{aligned}
& \Delta a\left(f_{0}, f\right)^{1 \mathrm{PN}}=-\frac{2 e \mu\left(\cos f-\cos f_{0}\right)\left[7+3 e^{2}+5 e\left(\cos f+\cos f_{0}\right)\right]}{c^{2}\left(1-e^{2}\right)^{2}}, \\
& \Delta e\left(f_{0}, f\right)^{1 \mathrm{PN}}=\frac{\mu\left(\cos f_{0}-\cos f\right)\left[3+7 e^{2}+5 e\left(\cos f+\cos f_{0}\right)\right]}{c^{2} a\left(1-e^{2}\right)} .
\end{aligned}
$$

The calculation of $\dot{\omega}_{\text {indir }}^{2 \mathrm{PN}}$ (II) by Kopeikin and Potapov [9] can be reproduced as follows [7] (p. 13). Evaluate the Gauss equation of the pericentre for a perturbing in-plane acceleration

$$
\frac{\mathrm{d} \omega}{\mathrm{d} f}=\frac{r^{2}}{\mu e}\left[-A_{\rho} \cos f+\left(1+\frac{r}{p}\right) \sin f A_{\tau}\right],
$$

where $p \doteq a\left(1-e^{2}\right)$, with the radial and transverse components of the 1PN acceleration of Equation (3)

$$
\begin{aligned}
& A_{\rho}^{1 \mathrm{PN}}=\frac{\mu^{2}(1+e \cos f)^{2}\left(3+e^{2}+2 e \cos f-2 e^{2} \cos 2 f\right)}{c^{2} a^{3}\left(1-e^{2}\right)^{3}}, \\
& A_{\tau}^{1 \mathrm{PN}}=\frac{4 e \mu^{2}(1+e \cos f)^{3} \sin f}{c^{2} a^{3}\left(1-e^{2}\right)^{3}} .
\end{aligned}
$$

Then, make the replacement

$$
\begin{aligned}
& a \rightarrow a+\Delta a\left(f_{0}, f\right)^{1 \mathrm{PN}} \\
& e \rightarrow e+\Delta e\left(f_{0}, f\right)^{1 \mathrm{PN}}
\end{aligned}
$$


by means of Equations (9) and (10), expand $\mathrm{d} \omega / d f$ to the order of $\mathcal{O}\left(c^{-4}\right)$, and integrate the resulting expression:

$$
\begin{aligned}
\left.\frac{\mathrm{d} \omega}{\mathrm{d} f}\right|_{\text {indir }} ^{2 \mathrm{PN}(\mathrm{II})} & =-\frac{\mu^{2}\left(\cos f-\cos f_{0}\right)}{2 c^{4} a^{2} e^{2}\left(1-e^{2}\right)^{2}}\left\{e\left[15-43 e^{2}+5\left(3+17 e^{2}\right) \cos 2 f\right]+\right. \\
& \left.+2 \cos f\left[9+48 e^{2}-e^{4}+5 e\left(3+e^{2}\right) \cos f_{0}\right]\right\}
\end{aligned}
$$

from $f_{0}$ to $f_{0}+2 \pi$. By dividing the result by $1 / P_{\mathrm{b}}=n_{\mathrm{b}} / 2 \pi$, the following formula is obtained:

$$
\dot{\omega}_{\text {indir }}^{2 \mathrm{PN}(\mathrm{II})}=\frac{n_{\mathrm{b}} \mu^{2}\left(-9-48 e^{2}+e^{4}-48 e^{3} \cos f_{0}\right)}{2 c^{4} a^{2} e^{2}\left(1-e^{2}\right)^{2}},
$$

so that the sum of Equations (4) and (17) gives the total indirect 2PN precession [7] (Equation (55))

$$
\dot{\omega}_{\text {indir }}^{2 \mathrm{PN}}=\frac{n_{\mathrm{b}} \mu^{2}\left(-11+2 e^{2}-48 e \cos f_{0}\right)}{2 c^{4} a^{2}\left(1-e^{2}\right)^{2}},
$$

which is, actually, correct. It turns out that summing Equation (18) to Equation (1) yields just the total 2PN precession of Equation (8).

Iorio [7] (p. 5) followed another approach to calculating $\dot{\omega}_{\text {indir }}^{2 \mathrm{PN}(\mathrm{II})}$. In the specific case of the pericentre and of Equation (3), starting from Equation(11), calculated with Equations (12) and (13), the net 2PN pericentre shift per orbit due to the instantaneous 1PN variations of Equations (9) and (10) is worked out as

$$
\Delta \omega_{\text {indir }}^{2 \mathrm{PN}(\mathrm{II})}=\int_{f_{0}}^{f_{0}+2 \pi}\left[\frac{\partial(\mathrm{d} \omega / \mathrm{d} f)}{\partial a} \Delta a\left(f_{0}, f\right)^{1 \mathrm{PN}}+\frac{\partial(\mathrm{d} \omega / \mathrm{d} f)}{\partial e} \Delta e\left(f_{0}, f\right)^{1 \mathrm{PN}}\right] \mathrm{d} f .
$$

A calculation error ${ }^{3}$ in the first addend of Equation (19) yielded the wrong result of Equation (5). After correcting it, it is possible to show that the function to be integrated in Equation (19) agrees with Equation (16). Thus, Equation (17) can also be correctly obtained with the method for calculating $\dot{\omega}_{\text {indir }}^{2 \mathrm{PN}}$ (II) $u$ sed by Iorio [7].

By repeating the calculation by Iorio [7], corrected for the aforementioned error, one obtains, for the full two-body system,

$$
\dot{\omega}_{\text {indir }}^{2 \mathrm{PN}}=\frac{n_{\mathrm{b}} \mu^{2}\left[-44+8 v(-8+7 v)+e^{2}\left(8+39 v+48 v^{2}\right)+96 e(-2+v) \cos f_{0}\right]}{8 c^{4} a^{2}\left(1-e^{2}\right)^{2}},
$$

which, summed to (Equation (32) in Iorio [7]), returns

$$
\dot{\omega}_{\mathrm{tot}}^{2 \mathrm{PN}}=\frac{3 n_{\mathrm{b}} \mu^{2}\left[2-4 v+e^{2}(1+10 v)+16 e(-2+v) \cos f_{0}\right]}{4 c^{4} a^{2}\left(1-e^{2}\right)^{2}} .
$$

In Equations (20) and (21), it is

$$
\begin{aligned}
& \nu \doteq \frac{M_{\mathrm{A}} M_{\mathrm{B}}}{\left(M_{\mathrm{A}}+M_{\mathrm{B}}\right)^{2}} \\
& \mu \doteq G\left(M_{\mathrm{A}}+M_{\mathrm{B}}\right) .
\end{aligned}
$$

In the limit $v \rightarrow 0$, Equation (21) agrees with Equation (8).

3 To be more specific, $\mu^{2}$ entering Equations (12)-(13) was expressed in $\mathrm{d} \omega / \mathrm{d} f$ as $n_{\mathrm{b}}^{4} a^{6}$, thus altering the partial derivative of $\mathrm{d} \omega / \mathrm{d} f$ with respect to $a$. 
It is now explicitly shown that (Kopeikin and Potapov [9] Equation (5.2)) coincides with our Equation (8) after the translation from one parameterisation to another is properly carried out. The starting point is, in the test particle limit, ([9] Equation (5.2))

$$
\frac{\Delta \omega_{\text {tot }}}{2 \pi}=\frac{3 \mu}{c^{2} k_{1}\left(1-k_{2}^{2}\right)}\left[1+\frac{3 \mu}{4 c^{2} k_{1}\left(1-k_{2}^{2}\right)}-\frac{\mu}{4 c^{2} k_{1}}\right] .
$$

Here, $k_{1}, k_{2}$ are the constants of integration ${ }^{4}$ of the solutions of the Gauss equations for the semimajor axis and the eccentricity to the 1PN level to be determined with the initial conditions at $t=t_{0}$. They can be obtained, e.g., by evaluating (Kopeikin and Potapov [9] Equations (4.5) and (4.6)) at $t=t_{0}$ by replacing $f$, i.e., $V$ in the notation by Kopeikin and Potapov [9], with $f_{0}$, and by recalling that, in our notation, $a_{0} \rightarrow k_{1}, e_{0} \rightarrow k_{2}$. Moreover, $a, e$ are, for us, the osculating numerical values of the semimajor axis and eccentricity, respectively, at the same arbitrary instant $t_{0}$; thus, $a\left(t_{0}\right), e\left(t_{0}\right)$ in the left-hand side of (Kopeikin and Potapov [9] Equations (4.5) and (4.6)) are just our $a, e$ here. Thus, in the limit $v \rightarrow 0$, one gets ${ }^{5}$

$$
\begin{aligned}
k_{1} & =\frac{a}{1+\frac{e \mu}{c^{2} a\left(1-e^{2}\right)^{2}}\left[\left(-14-6 e^{2}\right) \cos f_{0}-5 e \cos 2 f_{0}\right]} \simeq \\
& \simeq a+\frac{e \mu}{c^{2}\left(1-e^{2}\right)^{2}}\left[\left(14+6 e^{2}\right) \cos f_{0}+5 e \cos 2 f_{0}\right], \\
k_{2} & =\frac{e}{1+\frac{\mu}{c^{2} e^{2} a\left(1-e^{2}\right)}\left[\left(-3-7 e^{2}\right) e \cos f_{0}-\frac{5}{2} e^{2} \cos 2 f_{0}\right]} \simeq \\
& \simeq e+\frac{\mu}{2 c^{2} a\left(1-e^{2}\right)}\left[\left(6+14 e^{2}\right) \cos f_{0}+5 e \cos 2 f_{0}\right] .
\end{aligned}
$$

By inserting Equations (25) and (26) into Equation (24), multiplying by $n_{\mathrm{b}}$, and expanding the resulting expression in powers of $c^{-1}$ to the order $c^{-4}$, one just gets the sum of Equation (8) and of the 1PN precession:

$$
\dot{\omega}^{1 \mathrm{PN}}=\frac{3 n_{\mathrm{b}} \mu}{c^{2} a\left(1-e^{2}\right)} .
$$

As far as Mercury is concerned, for which it is [7] (Figure 1)

$$
\dot{\omega}_{\mathrm{dir}}^{2 \mathrm{PN}}=2.6 \mu \mathrm{ascty}^{-1} \text {, }
$$

where $\mu$ as cty ${ }^{-1}$ stands for microarcseconds per century, Equation (18) yields

$$
-4 \mu \mathrm{ascty}^{-1} \leq \dot{\omega}_{\text {indir }}^{2 \mathrm{PN}} \leq-0.2 \mu \text { as cty }^{-1},
$$

for $0^{\circ} \leq f_{0} \leq 360^{\circ}$; Equation (29) corrects (Iorio [7] (Equation (24)).

For the double pulsar PSR J0737-3039A/B, for which it is ([7] Equation (33))

$$
\dot{\omega}_{\mathrm{dir}}^{2 \mathrm{PN}}=0.00019^{\circ} \mathrm{yr}^{-1}
$$

4 Kopeikin and Potapov [9] used the notation $a_{0}, e_{0}$ instead of $k_{1}, k_{2}$.

5 A calculation error occurred in (Iorio [7] Equations (51) and (52)) for $k_{1}, k_{2}$. 
from Equation (20), which fives

$$
-0.00022^{\circ} \mathrm{yr}^{-1} \leq \dot{\omega}_{\text {indir }}^{2 \mathrm{PN}} \leq-0.00013^{\circ} \mathrm{yr}^{-1}
$$

for $0^{\circ} \leq f_{0} \leq 360^{\circ}$. For the Hulse-Taylor binary pulsar PSR B1913+16, for which it is [7] (Equation (35))

$$
\dot{\omega}_{\text {dir }}^{2 \mathrm{PN}}=0.000038^{\circ} \mathrm{yr}^{-1},
$$

Equation (20) yields

$$
-0.00009^{\circ} \mathrm{yr}^{-1} \leq \dot{\omega}_{\text {indir }}^{2 \mathrm{PN}} \leq 0.000034^{\circ} \mathrm{yr}^{-1}
$$

for $0^{\circ} \leq f_{0} \leq 360^{\circ}$. Equations (31) and (33) correct (Iorio [7] (Equations (48) and (49)).

For the supermassive binary black hole in OJ 287, for which it is [7] (p. 10)

$$
\dot{\omega}_{\mathrm{dir}}^{2 \mathrm{PN}}=11.0^{\circ} \mathrm{cty}^{-1},
$$

Equation (20) returns an indirect 2PN perinigricon precession ranging within

$$
-33.4^{\circ} \text { cty }^{-1} \leq \dot{\omega}_{\text {indir }}^{2 \mathrm{PN}} \leq 17^{\circ} \text { cty }^{-1}
$$

for $0^{\circ} \leq f_{0} \leq 360^{\circ}$. Equation (35) corrects the figures yielded in Iorio [7] (p. 12). In retrospect, they should have been a wake-up call concerning the validity of (Iorio [7] (Equation (47)), since the reported maximum value of $516^{\circ} \mathrm{cty}^{-1}$ is even larger than the 1PN precession itself, amounting to ([7] Equation (37)) $\dot{\omega}^{1 \mathrm{PN}}=206.8^{\circ} \mathrm{cty}^{-1}$.

The discussion in Iorio [7] concerning the measurability of the $2 \mathrm{PN}$ pericentre precessions of Mercury and of the binary pulsars will not be repeated here.

\section{Correcting the Error for $e_{\mathrm{T}}$}

As correctly pointed out by Kopeikin [11], Iorio [7] erroneously claimed that Equation (3.12) of Damour and Schafer [10]

$$
\frac{\Delta \omega_{\mathrm{tot}}^{2 \mathrm{PN}}}{2 \pi}=\frac{3}{c^{2} h^{2}}\left[1+\left(\frac{5}{2}-v\right) \frac{E}{c^{2}}+\left(\frac{35}{4}-\frac{5}{2} v\right) \frac{1}{c^{2} h^{2}}\right],
$$

and Equation (5.18) of Damour and Schafer [10]

$$
\begin{aligned}
\frac{\Delta \omega_{\text {tot }}^{2 \mathrm{PN}}}{2 \pi} & =\frac{3(\mu n)^{2 / 3}}{c^{2}\left(1-e_{\mathrm{T}}^{2}\right)}\left[1+\frac{(\mu n)^{2 / 3}}{c^{2}\left(1-e_{\mathrm{T}}^{2}\right)}\left(\frac{39}{4} x_{\mathrm{A}}^{2}+\frac{27}{4} x_{\mathrm{B}}^{2}+15 x_{\mathrm{A}} x_{\mathrm{B}}\right)-\right. \\
& \left.-\frac{(\mu n)^{2 / 3}}{c^{2}}\left(\frac{13}{4} x_{\mathrm{A}}^{2}+\frac{1}{4} x_{\mathrm{B}}^{2}+\frac{13}{3} x_{\mathrm{A}} x_{\mathrm{B}}\right)\right]
\end{aligned}
$$

would be mutually inconsistent after being expressed in terms of $a, e, f_{0}$. In Equations (36) and (37), $h$ and $E$ are the coordinate-invariant, reduced orbital angular momentum and energy, respectively,

$$
\begin{aligned}
& x_{\mathrm{A}} \doteq \frac{M_{\mathrm{A}}}{M_{\mathrm{A}}+M_{\mathrm{B}}}, \\
& x_{\mathrm{B}} \doteq \frac{M_{\mathrm{B}}}{M_{\mathrm{A}}+M_{\mathrm{B}}}=1-x_{\mathrm{A}},
\end{aligned}
$$

$n$ is the PN mean motion [14], and $e_{\mathrm{T}}$ is one of the several Damour-Deruelle (DD) parameters [15]. More precisely, in Iorio [7] (pp. 14-15), it was correctly demonstrated that Equation (36) yields Equation (8) once the typo of Equation (7) is corrected. On the 
other hand, in Iorio [7] (p. 15), it was erroneously claimed that Equation (37) could not reduce to Equation (8) due to the typo in Equation (7). The error consists of the fact that Iorio [7] confused $e_{\mathrm{T}}$ [15] entering Equation (37) with $e_{t}$, another member of the DD parameterisation [14].

Instead, it is [15] (p. 272)

$$
e_{\mathrm{T}}=e_{t}(1+\delta)+e_{\theta}-e_{r} .
$$

The parameters entering Equation (40) are defined as ([14] Equation (3.8b))

$$
e_{t}=\frac{e_{R}}{1+\frac{\mu}{c^{2} a_{R}}\left(4-\frac{3}{2} v\right)},
$$

([15] Equation (20))

$$
\delta=\frac{\mu}{c^{2} a_{R}}\left(x_{\mathrm{A}} x_{\mathrm{B}}+2 x_{\mathrm{B}}^{2}\right),
$$

([14] Equation (4.13))

$$
e_{\theta}=e_{R}\left(1+\frac{\mu v}{2 c^{2} a_{R}}\right)
$$

([14] Equation (6.3b))

$$
e_{r}=e_{R}\left[1-\frac{\mu}{2 c^{2} a_{R}}\left(x_{\mathrm{A}}^{2}-v\right)\right] .
$$

In Equations (41)-(44), the DD "semimajor axis" of the relative motion ${ }^{6} a_{R}$ can be expressed as ([7] Equation (66))

$$
\begin{aligned}
4\left(1-e^{2}\right)^{2} a_{R} & =4\left\{a\left(1-e^{2}\right)^{2}-\frac{\mu}{c^{2}}\left[-3+v+e^{4}(1+2 v)+e^{2}(-13+7 v)\right]\right\}+ \\
& +e \frac{\mu}{c^{2}}\left\{\left[56+e^{2}(24-31 v)-24 v\right] \cos f_{0}+\right. \\
& \left.+e\left[4(5-4 v) \cos 2 f_{0}-e v \cos 3 f_{0}\right]\right\},
\end{aligned}
$$

and the DD "eccentricity" $e_{R}$ is given by ${ }^{7}$ (Iorio [7] Equation (67)):

$$
\begin{aligned}
8 a\left(-1+e^{2}\right) e_{R} & =4 e\left\{2 a\left(-1+e^{2}\right)+\frac{\mu}{c^{2}}\left[-17+6 v+e^{2}(2+4 v)\right]\right\}+ \\
& +\frac{\mu}{c^{2}}\left\{\left[8(-3+v)+e^{2}(-56+47 v)\right] \cos f_{0}+\right. \\
& \left.+e\left[4(-5+4 v) \cos 2 f_{0}+e v \cos 3 f_{0}\right]\right\} .
\end{aligned}
$$

It turns out that, using Equations (40)-(46) and ([14] Equation (3.7))

$$
n=\sqrt{\frac{\mu}{a_{R}^{3}}}\left[1+\frac{\mu}{2 c^{2} a_{R}}(-9+v)\right]
$$

6 In Iorio [7], it is designed as $a_{r}$ : in fact, such a choice may be confusing, since, in (Damour and Deruelle [14] Equation (6.3 a)), such a quantity is meant as $a_{r}=M_{\mathrm{B}} a_{R} /\left(M_{\mathrm{A}}+M_{\mathrm{B}}\right)$.

7 In Iorio [7], it is denoted as $e_{r}$, but, in view of Equation (44), such a choice is misleading. 
in Equation (37) and expanding all to the order of $\mathcal{O}\left(c^{-4}\right)$ removes the previously mentioned alleged discrepancy. Indeed, now, the corresponding $2 \mathrm{PN}$ precession can be cast into the form of Equation (8) in the same way as in Equation (36).

\section{Some Considerations about the Occurrence of $f_{0}$ in the 2PN Pericentre Precession}

The fact that $f_{0}$ enters Equation (8) through Equation (17) appears to be a general feature of that part of the long-term orbital precessions arising from the inclusion of the instantaneous shifts of all the Keplerian orbital elements in performing the average of the right-hand side of the Gauss equation of any of them over an orbital period. Indeed, such a feature also explicitly occurs with the interplay of, e.g., the Newtonian acceleration due to the primary's quadrupole mass moment $J_{2}$ and Equation (3) in calculating (part of) the indirect rates of change of the Keplerian orbital elements of the order of $J_{2} / c^{2}$ by means of the Gauss equations, as done by ${ }^{8}$ Will [16], Iorio [20].

It should be remarked that Equation (8) does not pretend, by any means, to contain any new physics with respect to what was obtained earlier, with different computational approaches and different parameterisations, by Damour and Schafer [10] and Kopeikin and Potapov [9] in terms of first integrals of motion like $E$ and $h$. Indeed, as it was shown here, Equation (5.2) by Kopeikin and Potapov [9] and Equations (3.12) and (5.18) by Damour and Schafer [10] agree with Equation (8). The appearance of $f_{0}$ in Equation (8) is of a gauge nature, in that it does not influence the values of the integrals of motion for a fixed orbit. It should be remembered that, after all, in any practical data analysis of specific astronomical and astrophysical systems of interest, an underlying choice of a given parameterisation and gauge is always unavoidably made. In most cases, it generally corresponds to that adopted in the present study, as per International Astronomical Union (IAU) recommendations [21], which is just targeted to people aiming to practically design and/or perform tests using, say, Mercury, exoplanets close to their parent stars, or S-stars in Sgr A*. It should also be stressed that Equations (8) and (21) should not be thought of as something to be used in actual tests by straightforwardly comparing them to some directly measured pericentre precessions, since, depending on the specific astronomical scenario at hand, different approaches are practically used in reducing the observations in order to perform tests of gravitational theories. For example, in Solar System analyses, the General Theory of Relativity (GTR) is tested by modelling the propagation of electromagnetic waves, the dynamics of material bodies, and the functioning of measuring devices in a PN framework to a given order, as well as estimating, in a least-square sense, some dedicated parameters by fitting such models to a whole range of observations of different kinds that have been collected over a century or so. Instead, the data of binary pulsars are reduced with a timing formula $[15,22]$ rooted in the PN theoretical framework based on the DD parameterisation [14], which is not used in Solar System studies. In the case of the recent detection of the Schwarzschild precession (1PN) in the orbit of the S-star S2 [23], astrometric measurements of the stellar right ascension (RA) and declination (DEC) and spectroscopic observations of the star's radial velocity were used to extract it with a 14-parameter fit, including, among other things, the six parameters of the orbit and a dimensionless scaling parameter $f_{\mathrm{SP}}$ of Equation (27), which is 0 for Newton and 1 for GTR. Rather, Equations (8) and (21) should be interpreted, for a given astronomical system, as useful tools for quickly performing sensitivity analyses and error budgets starting from information that is usually available about it in order to preliminarily check if the 2PN effects are still far from the current or future experimental sensitivity, and if other competing dynamical features of motion are larger than them. The way the actual measurement would ultimately be accomplished is entirely another matter, which depends on the specific system at hand.

8 A terminological confusion may arise if one does not properly note that Will [16] used the adjective "mixed" to denote the PN-quadrupole acceleration of the order of $J_{2} / c^{2}$ [17-19] entering the equations of motion. Moreover, Will [16] dubbed as "cross-term effects" the contributions to the overall orbital precessions labeled as "indirect" or "mixed" by Iorio [20] and here. 


\section{Looking at Some of the Short-Period S-Stars in Sgr A*}

Recently, Peißker et al. [13] discovered some short-period S-stars orbiting the candidate supermassive black hole (SMBH) in the Galactic Center (GC) at Sgr A* [24,25]; their relevant orbital parameters are reported in Table 1.

Table 1. Key orbital parameters of some fast-revolving S-stars as per Table 2 of Peißker et al. [13]. Here, the semimajor axis $a$ is in milliparsecs (mpc), the orbital period $P_{\mathrm{b}}$ is in yr, and the eccentricity $e$ is dimensionless.

\begin{tabular}{cccc}
\hline Star & $\boldsymbol{a}(\mathbf{m p c})$ & $\boldsymbol{P}_{\mathbf{b}}(\mathbf{y r})$ & $\boldsymbol{e}$ \\
\hline S4714 & $4.079 \pm 0.012$ & $12.0 \pm 0.3$ & $0.985 \pm 0.011$ \\
S62 & $3.588 \pm 0.02$ & $9.9 \pm 0.3$ & $0.976 \pm 0.01$ \\
S4711 & $3.002 \pm 0.06$ & $7.6 \pm 0.3$ & $0.768 \pm 0.030$ \\
\hline
\end{tabular}

Since some of them can move as fast as $\simeq 10$ percent of the speed of light $c$ at perinigricon, it seems appropriate to look at their 2PN orbital effects. Recently, Peißker et al. [13], Fragione and Loeb [26], Iorio [27] investigated the 1PN gravitomagnetic LenseThirring orbital precessions induced by the SMBH's angular momentum $J_{\bullet}$ in view of a possible detection in the future.

Figure 1 displays the 2PN perinigricon precessions $\dot{\omega}^{2 \mathrm{PN}}$ and shifts per orbit $\Delta \omega^{2 \mathrm{PN}}$ of the S-stars S4711, S4714, and S62 as functions of $f_{0}$ according to Equation (8).

It turns out that the largest precessions, of the order of $\left|\dot{\omega}^{2 \mathrm{PN}}\right| \lesssim 4-6$ arcseconds per year (" $\left.\mathrm{yr}^{-1}\right)$, occur for S62 and S4714, whose shifts per orbit can be as large as $\left|\Delta \omega^{2 \mathrm{PN}}\right| \lesssim 40-90^{\prime \prime}$. Such figures are of about the same order of magnitude as the largest possible values of the 1PN Lense-Thirring precessions for $\omega$, as shown by Table 4 of Iorio [27]. Although future improvements in the observational techniques may possibly bring such post-Newtonian effects within the range of measurability [13], it should be remarked that they may be biased by the currently much larger systematic bias due to the lingering uncertainties in the stellar orbital parameters and the SMBH's mass entering the dominant 1PN component of the perinigricon precession of Equation (27); see Table 5 of Iorio [27]. Moreover, as a further source of systematic bias, it is also known that an extended mass distribution around the SMBH could concurrently affect the perinigricon precession [28-30]. 

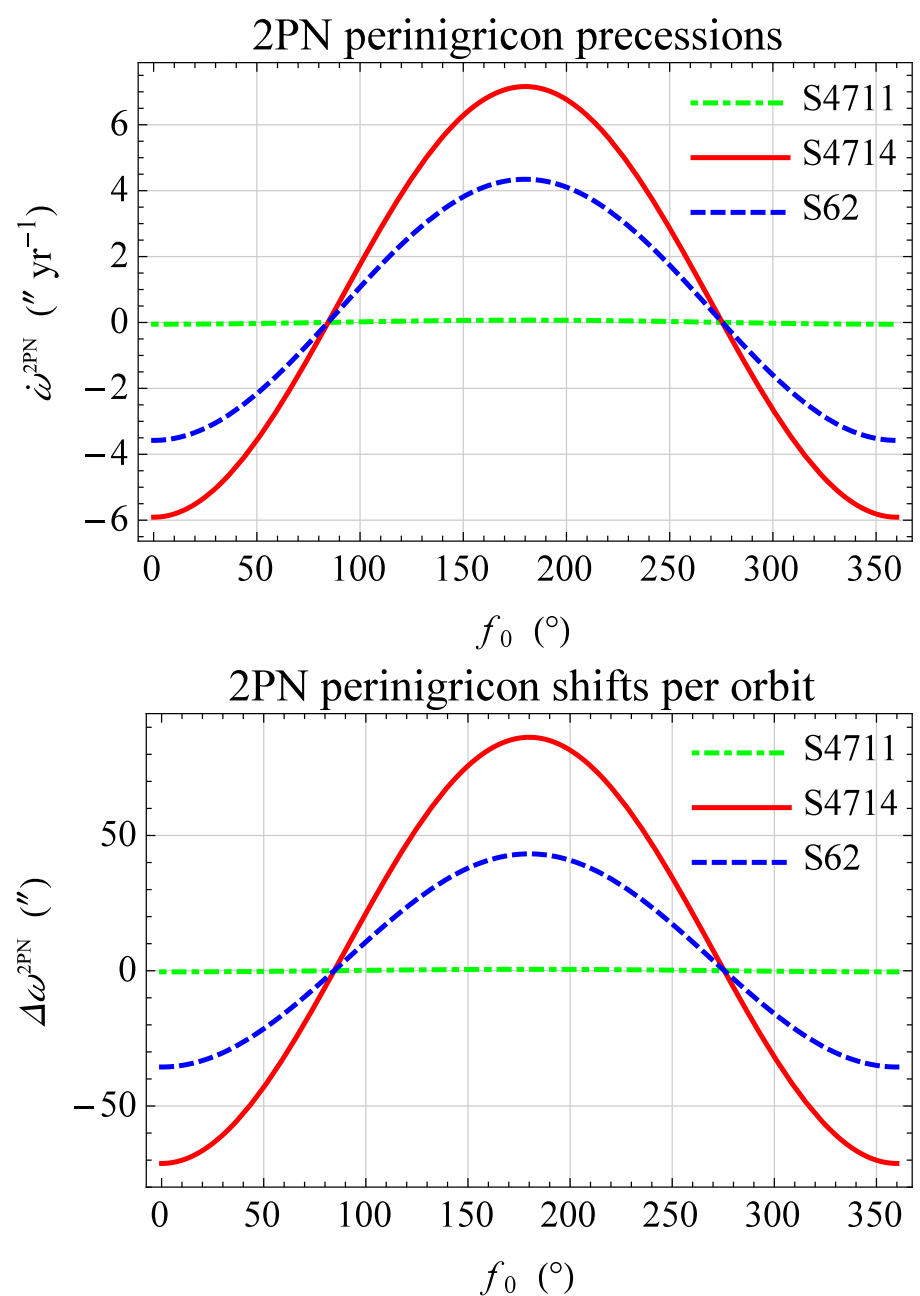

Figure 1. Upper panel: plot of the $2 \mathrm{PN}$ perinigricon precessions $\dot{\omega}^{2 \mathrm{PN}}$ of the S-stars S4711, S4714, and $\mathrm{S} 62$ [13] in arcseconds per year $\left({ }^{\prime \prime} \mathrm{yr}^{-1}\right)$ as a function of the true anomaly $f_{0}$ at the epoch according to Equation (8). Lower panel: plot of the $2 \mathrm{PN}$ perinigricon shifts per orbit $\Delta \omega^{2 \mathrm{PN}}=2 \pi / n_{\mathrm{b}} \dot{\omega}^{2 \mathrm{PN}}$ of the S-stars S4711, S4714, and S62 [13] in arcseconds (") as a function of the true anomaly $f_{0}$ at the epoch according to Equation (8).

\section{Summary and Conclusions}

After having disclosed and corrected the calculation errors affecting Iorio [7], it was demonstrated that the approaches by Iorio [7], Kopeikin and Potapov [9], Damour and Schafer [10] are, in fact, equivalent in analytically calculating the $2 \mathrm{PN}$ pericentre precession. Indeed, they yield the same result once the appropriate conversions from the adopted parameterisations are made. This demonstrates that the approach by Iorio [7] is correct.

The fact that the total $2 \mathrm{PN}$ pericentre rate of Equation (8) also contains $f_{0}$ due to the indirect contributions to it arising from the cross-coupling of the 1PN acceleration with itself in the calculation procedure is an example of a general feature that also seems to characterise other mixed effects due to the interplay of different accelerations (Newtonian and PN) in the orbital averaging of the Gauss equations when the instantaneous shifts of all the Keplerian orbital elements are also taken into account. The same feature also occurs, e.g., for the mixed precessions of the order of $J_{2} / c^{2}$ due to the Newtonian quadrupole acceleration and the $1 \mathrm{PN}$ one, as explicitly calculated by other authors in the literature. Nonetheless, this fact should not induce one to think about it as a sign of some new physics, as it is simply a matter of choice of a particular gauge. Choosing a given coordinate system and a gauge is a standard and unavoidable practice in actual data reductions and in designing possible future tests with astronomical and astrophysical systems of potential interest. 
To this aim, Equation (8) was used to calculate the magnitude of the 2PN perinigricon precessions for some of the recently discovered fast-orbiting S-stars in Sgr A*, whose orbital configuration is given in the literature, in terms of the osculating Keplerian orbital elements adopted here. It turns out that the shifts per orbit of S62 and S4714 can be as large as $\simeq 40-90$ arcseconds, as they are, in principle, detectable in view of the expected progress in the observational techniques. Nonetheless, a major source of systematic bias lies in the lingering uncertainty in the much larger $1 \mathrm{PN}$ component of the stellar perinigricon rates.

Funding: This research received no external funding.

Acknowledgments: I am grateful to M. Efroimsky for the useful advice.

Conflicts of Interest: The authors declare no conflict of interest.

\section{References}

1. Blanchet, L. Post-Newtonian Theory and its Application. In Proceedings of the Twelfth Workshop on General Relativity and Gravitation in Japan; Shibata, M., Eriguchi, Y., Taniguchi, K., Nakamura, T., Tomita, K., Eds.; The University of Tokyo: Komaba, Tokyo, 2003; pp. 8-23.

2. Will, C.M. The Confrontation between General Relativity and Experiment. Living Rev. Relativ. 2014, 17, 4. [CrossRef] [PubMed]

3. Will, C.M. Theory and Experiment in Gravitational Physics, 2nd ed.; Cabridge University Press: Cambridge, UK, 2018.

4. Zhang, X.; Zhao, W.; Liu, T.; Lin, K.; Zhang, C.; Zhao, X.; Zhang, S.; Zhu, T.; Wang, A. Constraints of General Screened Modified Gravities from Comprehensive Analysis of Binary Pulsars. Astrophys. J. 2019, 874, 121. [CrossRef]

5. Kopeikin, S.; Efroimsky, M.; Kaplan, G. Relativistic Celestial Mechanics of the Solar System; Wiley-VCH: Weinheim, Germany, 2011. [CrossRef]

6. Soffel, M.H.; Han, W.B. Applied General Relativity; Astronomy and Astrophysics Library, Springer Nature Switzerland: Cham, Switzerland, 2019.

7. Iorio, L. Revisiting the 2PN Pericenter Precession in View of Possible Future Measurements. Universe 2020, 6, 53. [CrossRef]

8. Klioner, S.A.; Kopeikin, S.M. The Post-Keplerian Orbital Representations of the Relativistic Two-Body Problem. Astrophys. J. 1994, 427, 951. [CrossRef]

9. Kopeikin, S.M.; Potapov, V.A. Relativistic shift of the periastron of a double pulsar in the post-post-Newtonian approximation of General Relativity. Astron. Rep. 1994, 38, 104-114.

10. Damour, T.; Schafer, G. Higher-order relativistic periastron advances and binary pulsars. Nuovo Cimento B 1988, 101, 127-176. [CrossRef]

11. Kopeikin, S.M. The Orbital Pericenter Precession in the 2PN Approximation. Eur. J. Phys. Plus 2020, 135, 466. [CrossRef]

12. Amaro-Seoane, P. The gravitational capture of compact objects by massive black holes. In Handbook of Gravitational Wave Astronomy; Bambi, C., Katsanevas, S., Kokkotas, K., Eds.; Springer: Singapore, 2021; at press.

13. Peißker, F.; Eckart, A.; Zajaček, M.; Ali, B.; Parsa, M. S62 and S4711: Indications of a Population of Faint Fast-moving Stars inside the S2 Orbit-S4711 on a 7.6 yr Orbit around Sgr A*. Astrophys. J. 2020, 899, 50. [CrossRef]

14. Damour, T.; Deruelle, N. General relativistic celestial mechanics of binary systems. I. The post-Newtonian motion. Ann. Inst. Henri Poincaré Phys. Théor. 1985, 43, 107-132.

15. Damour, T.; Deruelle, N. General relativistic celestial mechanics of binary systems. II. The post-Newtonian timing formula. Ann. Inst. Henri Poincaré Phys. Théor 1986, 44, 263-292.

16. Will, C.M. Incorporating post-Newtonian effects in N-body dynamics. Phys. Rev. D 2014, 89, 044043. [CrossRef]

17. Soffel, M.; Wirrer, R.; Schastok, J.; Ruder, H.; Schneider, M. Relativistic effects in the motion of artificial satellites. I-The oblateness of the central body. Celest. Mech. Dyn. Astr. 1987, 42, 81-89. [CrossRef]

18. Soffel, M.H. Relativity in Astrometry, Celestial Mechanics and Geodesy; Springer: Berlin/Heidelberg, Germany, 1989.

19. Brumberg, V.A. Essential Relativistic Celestial Mechanics; Adam Hilger: Bristol, UK, 1991.

20. Iorio, L. Post-Newtonian direct and mixed orbital effects due to the oblateness of the central body. Int. J. Mod. Phys. D 2015, 24, 1550067-59. [CrossRef]

21. Soffel, M.; Klioner, S.A.; Petit, G.; Wolf, P.; Kopeikin, S.M.; Bretagnon, P.; Brumberg, V.A.; Capitaine, N.; Damour, T.; Fukushima, T.; et al. The IAU 2000 Resolutions for Astrometry, Celestial Mechanics, and Metrology in the Relativistic Framework: Explanatory Supplement. Astron. J. 2003, 126, 2687-2706. [CrossRef]

22. Wex, N. Testing relativistic gravity with radio pulsars. In Frontiers in Relativistic Celestial Mechanics. Volume 2 Applications and Experiments; De Gruyter Studies in Mathematical Physics; Kopeikin, S., Ed.; de Gruyter: Berlin, Germany; Boston, MA, USA, 2014; Volume 22, pp. 39-102.

23. Gravity Collaboration; Abuter, R.; Amorim, A.; Bauböck, M.; Berger, J.P.; Bonnet, H.; Brandner, W.; Cardoso, V.; Clénet, Y.; de Zeeuw, P.T.; et al. Detection of the Schwarzschild precession in the orbit of the star S2 near the Galactic centre massive black hole. Astron. Astrophys. 2020, 636, L5, 
24. Ghez, A.M.; Salim, S.; Weinberg, N.N.; Lu, J.R.; Do, T.; Dunn, J.K.; Matthews, K.; Morris, M.R.; Yelda, S.; Becklin, E.E.; et al. Measuring Distance and Properties of the Milky Way's Central Supermassive Black Hole with Stellar Orbits. Astrophys. J. 2008, 689, 1044-1062. [CrossRef]

25. Genzel, R.; Eisenhauer, F.; Gillessen, S. The Galactic Center massive black hole and nuclear star cluster. Rev. Mod. Phys. 2010, 82, 3121-3195. [CrossRef]

26. Fragione, G.; Loeb, A. An Upper Limit on the Spin of SgrA* Based on Stellar Orbits in Its Vicinity. Astrophys. J. Lett. 2020, 901, L32. [CrossRef]

27. Iorio, L. The short-period S-stars S4711, S62, S4714 and the Lense-Thirring effect due to the spin of Sgr A*. Astrophys. J. 2020, 904, 186. [CrossRef]

28. Rubilar, G.F.; Eckart, A. Periastron shifts of stellar orbits near the Galactic Center. Astron. Astrophys. 2001, 374, 95-104. [CrossRef]

29. Nucita, A.A.; De Paolis, F.; Ingrosso, G.; Qadir, A.; Zakharov, A.F. Sgr A*: A Laboratory to Measure the Central Black Hole and Stellar Cluster Parameters. Publ. Astron. Soc. Pac. 2007, 119, 349-359. [CrossRef]

30. Zakharov, A.F.; Nucita, A.A.; de Paolis, F.; Ingrosso, G. Apoastron shift constraints on dark matter distribution at the Galactic Center. Phys. Rev. D 2007, 76, 062001. [CrossRef] 